\title{
Editorial: Mapping the visual brain
}

According to Ptolemy (90-168), the father of modern cartography, the fundamental task of the mapmaker is "to describe the whole in its just proportions." Contemporary neuroscientists surveying the serial, parallel, hierarchical and interconnected pathways that comprise the neural substrates of vision have discovered and charted a bewildering array of representations of the visual field in the vertebrate brain, yet the whole of this system is yet to be fully described and conceptualized. The central question remains: Why are there so many maps and what are they all doing?

Numerous comparative studies have demonstrated that a consistent pattern of first-order retinorecipient targets exists across diverse taxonomic groups, in support of the notion that a fundamental and phylogenetically ancient "bauplan" for vision occurs in all extant vertebrates. To date, some 9-12 firstorder targets of retinal axons have been identified and charted, with only the hypothalamic and accessory optic projections seeming to lack a well-defined retinotopic organization. The extent to which the coordinates of these early maps are related to retinal ganglion-cell densities and whether the scale for central vs. peripheral, superior vs. inferior, or nasal vs. temporal representations are the same across different retinorecipient nuclei has not been fully assessed for any species. Although each individual map is quadratically and locally ordered, it also may be globally distorted in accordance with the functional significance of that particular pathway.

Second-order optic projections that interconnect the primary retinorecipient nuclei may be topographically organized as well, even though the coordinates of the interconnected maps are not always the same, thus raising important questions about the ontogenetic mechanisms that underlie the development of topographic correspondence. Neural representations of the retina may be further parcellated into distinct maps, even within the same projection pathway, by morphologically and functionally distinct subpopulations of neurons (X \& Y, P \& M) which may have different magnification and visuotopic scaling factors in striate and extrastriate cortical fields. Ultimately, cortical areas specialized for the analysis of specific features and/or stimulus dimensions may receive convergent input from physiologically distinct pathways that remain segregated at earlier stages in the visual system.

At last count, more than 30 areas and over 300 interconnect- ing pathways have been identified that are associated with some aspect of vision in primate cortex, yet the central question remains: Why are there so many maps and what are their functional correlates? The hypothesis of cortical modularity, that the cortex is structurally and functionally organized into discrete modules which interact to produce cognitive and mental processes, will be thoroughly tested both empirically and computationally in this decade. The application of neuroimaging techniques to functional mapping in the intact brain - positron emission tomography, magnetic resonance imaging, magnetoelectroencephalography, and high-resolution optical imaging should dramatically advance our understanding of the sequential and spatial patterns of activation that are associated with specific visuomotor tasks and visual perception.

Equally promising are recent demonstrations based upon responses recorded from single, cortical neurons in trained, awake monkeys showing that perceptual decisions can be closely correlated with the activity of a small number of neurons (perhaps only one!). Such analyses lead. us ever closer to understanding the mechanisms whereby cortical signals modulate motoric and cognitive events. An opposing viewpoint, the population-coding hypothesis, postulates that perception and visuomotor behaviors depend upon patterns of distributed activity occurring in large populations or networks of neurons that correspond to an internal representation of the visual world. This formulation is not necessarily incompatible with the view that individual cortical areas may be preferentially, but not exclusively, committed to specific stimulus features such as motion, color, stereopsis, spatial location, etc. Indeed, selective visual attention can profoundly affect and alter the responses of single neurons to different attributes of the visual stimulus within the same cortical area, and the encoding of invariant or more global features of objects and patterns at successively higher levels in the cortical mosaic may also occur even within a single cortical area.

Neural cartography will continue to provide major impetus at the forefront of investigations into the organization of neural representations of the external world and the ways in which visuomotor action, visual perception and cognitive processes are generated within the complex circuity of the visual brain.

Katherine V. Fite Editor 